\title{
BOUNDED MATRICES AND SOME ALGEBRAS OF OPERATORS
}

\author{
DONAL P. O'DONOVAN
}

\begin{abstract}
A necessary and sufficient condition for a matrix to represent a continuous operator is given. Then a natural family of algebras of bounded operators is introduced.
\end{abstract}

This paper consists of two parts. The first part is simply the observation that the condition for the continuity of a Laurent or Toeplitz matrix, namely that the "function" be essentially bounded, is in a general sense true for any matrix. This appears not to have been widely realised, and since it is of some interest for its own sake we have presented it separately.

The remainder of the paper takes the analogy suggested by the first part and shows how it leads to a certain natural family of subalgebras of the bounded operators.

1. Bounded matrices. Let $\left\{e_{k}\right\}_{k=0}^{\infty}$ be an orthonormal basis for a separable Hilbert space $H$. Let $P_{n}$ denote the orthogonal projection on the span of $\left\{e_{k}\right\}_{k=0}^{n}$. The simplest necessary and sufficient condition for a linear map $A: H \rightarrow H$ to be bounded or continuous is that the operator norms of $\left\{P_{n} A P_{n}\right\}$ be uniformly bounded. This is just $\left\|P_{n} A P_{n}\right\| \leqslant\|A\|, \forall n$ and that $P_{n} A P_{n}$ converges weakly to $A$. If we denote the set of bounded operators by $L(H)$, we may define for all $k \in Z, E_{k}(A) \in L(H)$, by $\left(E_{k}(A) e_{n}, e_{m}\right)=\left(A e_{n}, e_{m}\right)$ - $\delta_{n-m, k}$, where $\delta_{n-m, k}$ is the usual Kronecker delta. For $A$ to be bounded it is a sufficient condition that $\left\{\sum_{k=-n}^{n} E_{k}(A)\right\}$ be uniformly bounded, as weak convergence will verify. However a consideration of Toeplitz operators shows that it is not necessary. But if we define $\sigma_{k}(A) \in L(H)$, by

$$
\sigma_{k}(A)=\sum_{j=-k}^{k} \frac{k-|j|}{k} E_{j}(A),
$$

the $k$ th Cesàro mean of $\left\{E_{k}(A)\right\}$, then

Theorem. $\|A\|=\sup _{k}\left\|\sigma_{k}(A)\right\|$.

Proof. If $\left\{\sigma_{k}(A)\right\}$ is uniformly bounded then $\sigma_{k}(A)$ converges weakly to $A$. It follows that $\|A\| \leqslant \sup _{k}\left\|\sigma_{k}(A)\right\|$.

Conversely, for all complex $\lambda \in S^{1}=\{\lambda:|\lambda|=1\}$, define the diagonal unitary operator $U_{\lambda}$ by $U_{\lambda} e_{k}=\lambda^{k} e_{k}$. For $\xi, \eta$ in $H,\|\xi\|=\|\eta\|=1$ define 
$f_{\xi, \eta}(\lambda)=\left(A U_{\lambda} \xi, U_{\lambda} \eta\right)$. Then $A \in L(H)$ implies $f_{\xi, \eta}$ is in $L^{\infty}\left(S^{1}\right)$.

If $\xi=\sum_{0}^{\infty} r_{n} e_{n}, \eta=\sum_{0}^{\infty} s_{n} e_{n} ; r_{n}, s_{n} \in \mathbf{C}$, then rearranging the double summation we have

$$
f_{\xi, \eta}(\lambda)=\sum_{k=-\infty}^{\infty}\left(\sum_{n-m=k} r_{n} \bar{s}_{m}\left(A e_{n}, e_{m}\right)\right) \cdot \lambda^{k}
$$

at least formally.

Suppose for the moment that (1.1) has been shown to be the actual Fourier expansion for $f_{\xi, \eta}(\lambda)$ as an element of $L^{2}\left(S^{1}\right)$. Then since $f_{\xi, \eta}$ is in $L^{\infty}\left(S^{1}\right)$, the Cesàro means of its Fourier expansion are uniformly bounded by $\left\|f_{\xi, \eta}\right\|_{\infty}[4$, p. 23]. Thus

$$
\sup _{|\lambda|=1}\left\|\sigma_{k}\left(f_{\xi, \eta}(\lambda)\right)\right\| \leqslant\left\|f_{\xi, \eta}\right\|_{\infty} \leqslant\|A\| \quad \text { for all } k .
$$

Now

$$
\left|\left(\sigma_{k}(A) U_{\lambda} \xi, U_{\lambda} \eta\right)\right|=\left|\sum_{j=-k}^{k} \frac{k-|j|}{k} \sum_{n-m=j}\left(A e_{n}, e_{m}\right) \cdot r_{n} \bar{s}_{m} \cdot \lambda^{j}\right|
$$

again at least formally

$$
=\left|\sigma_{k}\left(f_{\xi, \eta}(\lambda)\right)\right| \text {, from }(1.1) \text {. }
$$

Hence for

$$
\begin{aligned}
\|\xi\| & =\|\eta\|=1, \\
\left\|\sigma_{k}(A)\right\| & =\sup _{\xi, \eta}\left|\left(\sigma_{k}(A) \xi, \eta\right)\right|=\sup _{\xi, \eta}\left|\left(\sigma_{k}\left(f_{\xi, \eta}(\lambda)\right)\right)\right| \leqslant\|A\| .
\end{aligned}
$$

It remains to show that (1.1) and (1.2) are in fact the Fourier expansions. But $P_{n} A P_{n} \stackrel{w}{\longrightarrow} A$, so that $f_{\xi, \eta}^{n}(\lambda)=\left(P_{n} A P_{n} U_{\lambda} \xi, U_{\lambda} \eta\right) \rightarrow f_{\xi, \eta}(\lambda)$, each $\lambda$, and $\left\|f_{\xi, \eta}^{n}\right\|_{\infty} \leqslant\|A\|$ for all $n$. It follows that the sequence $\left\{f_{\xi, \eta}^{n}\right\}$ converges to $f_{\xi, \eta}$ in $L^{2}$ norm and then that the individual Fourier coefficients converge. An examination of the expansion of $f_{\xi, \eta}^{n}$, which is finite, gives (1.1). Replacing $A$ by $\sigma_{k}(A)$ gives (1.2).

REMARK 1. If one defines $f_{\xi, \eta}(\lambda)=\left(A U_{\lambda} \xi, U_{\bar{\lambda}} \eta\right)$ one obtains results analogous to those on Hankel matrices [3], [5]. Taking $f_{\xi, \eta}(\lambda)=\left(A U_{\lambda} \xi, \eta\right)$ or $(A \xi$, $\left.U_{\lambda} \eta\right)$ is another obvious variation.

REMARK 2. In any of these cases, given any collection of orthogonal projections with $\sum Q_{n}=1$, we can put $U_{\lambda} Q_{n}=\lambda^{n} \cdot Q_{n}$. Again the machine can be cranked, most naturally of course, if all the $Q_{n}$ have the same dimension.

2. Some algebras of operators. The basis chosen for $H$ in the last section was indexed by $Z^{+}$. The situation is identical, mutatis mutandi, for a two-sided basis indexed by $Z$. In this section we shall assume such a basis. Now the result of the last section is that $L(H)$ has a certain similarity to $L^{\infty}\left(S^{1}\right)$. In fact in [1], W. B. Arveson constructs expectations from $L(H)$ onto both the Laurent and Toeplitz operators.

Let $E_{k}(A)$ be defined, as earlier, for all $A$ in $L(H)$. Each $E_{k}$ is clearly 
continuous and linear. Let $\tilde{C}$ be the ${ }^{*}$-algebra consisting of all operators $A$ in $L(H)$ for which $E_{k}(A)=0$ for all but finitely many $k$. Let $C$ be the $C^{*}$ algebra which is the norm closure of $\tilde{C}$. Let $U$ be the bilateral shift operator defined by $U e_{n}=e_{n+1}, n \in Z$, and let $D$ denote the $C^{*}$-subalgebra of $L(H)$ consisting of all operators which are diagonal with respect to $\left\{e_{n}\right\}_{n \in Z}$. Let the action of $Z$ on $D$ be given by $U$, i.e. $B \rightarrow U B U^{*}$, then $C$ is in fact the semidirect product or covariance algebra $C^{*}(D, Z)$ [6], [9], and $C$ behaves like $C\left(S^{1}\right)$, the continuous functions, in the sense that each element $A$ is the norm limit of its Cesàro means $\sigma_{k}(A)$ defined earlier. This last fact was first pointed out to me by W. B. Arveson. We may obtain a subalgebra $T$ of $L(H)$ analogous to $H^{\infty}+C$ [2], [8], by taking

$$
T=\left\{A: E_{k}(A)=0\left(\forall k<\text { some } k_{0}\right)\right\}^{-}
$$

where - denotes the norm closure. Now putting $C_{Q}=T \cap T^{*}$ we obtain a $C^{*}$-subalgebra of $L(H)$ analogous to the quasicontinuous functions $H^{\infty}$ $+C \cap \overline{H^{\infty}+C}$.

If $\mathfrak{A}$ is any $C^{*}$-subalgebra of $D$, invariant under the previously described action of $Z$, implemented by $U$, say, then

$$
C(\mathfrak{U})=\left\{A: E_{k}(A)=0 \text { if } k \leqslant k_{0} \text { and } k \geqslant k_{1} \text {, some } k_{0}, k_{1},\right.
$$

$$
\text { and } \left.E_{k}(A) \in \mathfrak{A} U^{k} \text { for all } k\right\}^{-}
$$

is still a $C^{*}$-algebra. In fact $C(\mathfrak{U})=C^{*}(\mathfrak{A}, Z)$. But $\left\{A: E_{k}(A)\right.$ $\in \mathfrak{A} U^{k}$ for all $\left.k\right\}$ is not necessarily an algebra unless $\mathfrak{A}$ (which is a commutative $C^{*}$-algebra) has a totally disconnected spectrum. This is because one may not have $E_{k}\left(A_{1} \cdot A_{2}\right) \in \mathfrak{A} U^{k}$. However, we do have

Lemma. For any $\mathfrak{A}$, an invariant subalgebra of $D, C_{Q}(\mathfrak{H})=\left\{A: A \in C_{Q}\right.$, and $E_{k}(A) \in \mathfrak{A} U^{k}$ for all $\left.k\right\}$ is a $C^{*}$-algebra.

Proof. Let $A, B$ be given in $C_{Q}(\mathfrak{A})$. Approximate $A$ and $B$ by sequences $\left\{A_{n}\right\}$ and $\left\{B_{n}\right\}$ with the property that $E_{k}\left(A_{n}\right)=E_{k}\left(B_{n}\right)=0$ if $k \geqslant j_{n}$. Each $E_{k}\left(A_{n} \cdot B_{n}\right)$ is the sum of a finite number of elements of $\mathfrak{Y} U^{k}$, and the continuity of each $E_{k}$ completes the argument.

Thus we have a large family of $C^{*}$-algebras whose structure compares to that of a covariance algebra as the quasicontinuous functions compare to the continuous ones. Now R. G. Douglas showed that $H^{\infty}+C$ is simply one of the family of algebras $\Sigma_{\Lambda}$, determined from a semigroup $\Lambda$ of inner functions by

$$
\Sigma_{\Lambda}=\left\{\bar{\chi} \phi: \chi \in \Lambda, \phi \in H^{\infty}\right\}^{-}
$$

With $T$ as previously defined, and $L_{\chi}$ the Laurent operator associated with the function $\chi$, let

$$
T_{\Lambda}=\left\{L_{\chi}^{*} \cdot B: B \in T, \chi \in \Lambda\right\} \text { and } C_{Q, \Lambda}=T_{\Lambda} \cap T_{\Lambda}^{*} .
$$


If $\Lambda$ consists only of continuous inner functions, then we can add to $T_{\Lambda}$ the additional condition that each $E_{k}(A) U^{-k}$ belong to some given invariant $C^{*}$ algebra $\mathfrak{A}$. If not, then as before, we must restrict to those cases with totally disconnected spectrum. Together with requiring $\mathfrak{A}$ to be invariant, this reduces one to $\mathfrak{A}=D_{k_{i}}, 1 \leqslant k_{i} \leqslant \infty$, where $D_{k_{i}}$ is the subalgebra of all periodic diagonals whose period divides $k_{i}$.

If the analogy between these subalgebras of $L(H)$ and the corresponding subalgebras of $L^{\infty}\left(S^{1}\right)$ is as complete as it seems, one would expect $T_{\Lambda_{\infty}}$ to actually be $L(H)$, where $\Lambda_{\infty}$ is the semigroup of all inner functions. This appears likely but we have been unable to verify it.

Again we point out that the definitions we have given make perfectly good sense for a one-sided basis. A minor inconvenience is that $C$ is no longer a covariance algebra, but it behaves quite similarly. In particular the uniform convergence of the Cesàro means is still true.

One particularly interesting example is furnished by taking $\mathfrak{A}$ to be all compact diagonals. Then $C(\mathfrak{U})$ is $C(H)$, the $C^{*}$-algebra of all compact operators and $C_{Q}(\mathfrak{A})$ also has a simple interpretation. With $P_{n}$ as in the first section, it is shown in $[1, \S 2]$ that every operator which is quasitriangular with respect to these $P_{n}$ 's, i.e. $\left\|\left(1-P_{n}\right) A P_{n}\right\| \rightarrow 0$, is of the form triangular + compact. However, J. Plastiras [7] has shown that the quasidiagonal operators $\left\|P_{n} A-A P_{n}\right\| \rightarrow 0$ are not all of the form diagonal + compact.

THEOREM. Every such quasidiagonal operator is of the form diagonal + an element of $C_{Q}(\mathfrak{A})$.

Proof. $A$ is quasitriangular, so by W. B. Arveson's result $A$ is of the form triangular + compact. But every compact is the uniform limit of its Cesàro means, so $A \in T$, which was defined earlier. Similarly, $A^{*} \in T$, so $A \in C_{Q}$. But

$$
\left(A e_{n}, e_{n+k}\right)= \begin{cases}\left(\left(1-P_{n}\right) A P_{n} e_{n}, e_{n+k}\right) & \text { if } k>0 \\ \left(P_{n+k} A\left(1-P_{n+k}\right) e_{n}, e_{n+k}\right) & \text { if }-n<k<0\end{cases}
$$

so the hypothesis implies that for $k \neq 0,\left(A e_{n}, e_{n+k}\right) \rightarrow 0$, i.e. $E_{k}(A) \in \mathfrak{A}$ - $U^{k}$, and we have the desired conclusion. Here $U$ is the unilateral shift.

It would be of interest to determine whether others of the algebras $C_{Q, \Lambda}(\mathfrak{U})$ can be given a similarly concrete interpretation.

\section{REFERENCES}

1. W. B. Arveson, Interpolation problems in nest algebras (preprint).

2. R. G. Douglas, On the spectrum of Toeplitz and Wiener-Hopf operators, Abstract Spaces and Approximation (Proc. Conf., Oberwolfach, 1968), Birkhäuser, Basel, 1969, pp. 53-66. MR 41 \#4274.

3. P. Hartman, On completely continuous Hankel matrices, Proc. Amer. Math. Soc. 9 (1958), 862-866. MR 21 \# 7399.

4. K. Hoffman, Banach spaces of analytic functions, Prentice-Hall Ser. in Modern Analysis, Prentice-Hall, Englewood Cliffs, N. J., 1962. MR 24 \# A2844.

5. Z. Nehari, On bounded bilinear forms, Ann. of Math. (2) 65 (1957), 153-162. MR 18, 633. 
6. D. P. O'Donovan, Weighted shifts and covariance algebras, Trans. Amer. Math. Soc. 208 (1975), 1-25.

7. J. Plastiras, Compact perturbations of $C^{*}$-algebras, Dissertation, University of California, Berkeley, 1975.

8. D. Sarason, Generalized interpolation in $H^{\infty}$, Trans. Amer. Math. Soc. 127 (1967), 179-203. MR 34 \#8193.

9. G. Zeller-Meier, Produits croisés d'une $C^{*}$-algèbre par un groupe d'automorphismes, J. Math. Pures Appl. (9) 47 (1968), 101-239. MR 39 \#3329.

Department of Mathematics, Dalhousie University, Halifax, Nova Scotia, Canada 